\title{
Traditional and experimental aspects of cognitive design mechanisms
}

\author{
Irina Kirienko ${ }^{1, *}$, Tatiana Makhova ${ }^{1}$, and Viktor Piirainen ${ }^{2}$ \\ ${ }^{1}$ Sochi State University, Sochi, Russia \\ ${ }^{2}$ Saint-Petersburg Mining University, Saint-Petersburg, Russia
}

\begin{abstract}
The article considers the possibilities of the language of spatial forms that express various emotional states. The design of the urban-resort environment of Sochi becomes a carrier of a certain mood, which makes it figuratively significant. Through cognitive mechanisms, a person is included in the dialogue of reading the environmental text and the allusions, symbols, metaphors contained in it, etc. In an ecological design, the mechanism of synthesis of traditional and experimental aspects is laid down, which are embodied in a form that is accessible to perception through its emotional qualities, which affect consciousness through the synergistic influence of form and content.
\end{abstract}

\section{Introduction}

The semantics and symbolism of the regional landscape of the resort city of Sochi determine the design method of local environmental ensembles as the main tool in shaping the expressiveness of a unique regional environment. Creative project reconstruction of an unbalanced urban environment encourages the formation of a synthetic mythologized project space, thereby providing metaphorical images of an individual vision.

"We do not understand art if we do not feel in it the desire to experience the spiritual at first, at least in the revelation of a beautiful illusion" (R. Steiner) [1]. Metaphorical images of individual vision are implemented in the design of the urban environment-the traditional and experimental aspects of educational design at the Department of Architecture, Design and Ecology of the Sochi State University. Partial compensation for the loss of environmental unity is provided in the undivided teaching of theory and practice, that is, in the teaching of the environment. The theoretical training focuses on regional features for creative project reconstruction, thus ensuring a high level of creative thinking of students.

The initial position of the conducted research is the position that when solving creative tasks, the main thing is the ability to analyse and summarize the experience of activity in specific environmental conditions, taking into account historical traditions and modern requirements for material culture. The development of the art and design project language based on the traditions of continuity is manifested in the metaphorical transfer of the features of the regional natural environment to the design objects. At the same time,

\footnotetext{
* Corresponding author: 1 t_zhukova@mail.ru
} 
students, being introduced to experimental creative thinking, master the logic of traditional project thinking: "the influence of architectural and design forms on consciousness, knowledge of the world through the synergistic influence of form and content" [2].

\section{Materials and methods}

Significant changes in traditional design approaches are based on the search for original ideas. The initial requirement for experimental design is a comprehensive account of the technogenic and natural conditions of the design object, their correlation with historical and cultural features, traditions, as well as an assessment of the possibility of using the sensory contact with nature necessary for a person as a mechanism of cognitive attitude to design.

Based on this, the purpose of the study is to develop an environmentally sound model of the urban - resort environment of Sochi in the "person-landscape-environment" system.

In accordance with this goal, the following tasks were set:

1. Analyse the concepts of environmental design-landscape-environmental, subjectspatial, and structural-figurative approaches in the system of traditional aspect.

2. To analyse the concept of experimental design as a search approach to emphasizing the tasks of recreating the lost. At the same time, the attitude of self-perception as a part of the studied or projected environmental whole, cognitive identification with it, develops.

3. To develop a model of cognitive mechanisms of design from the perspective of expanding the emotional palette of the urban resort environment.

\section{Experimental results}

In the existing natural conditions of the region, the possibilities of many variations are laid down, which can be used in the construction of experimental alternative design solutions. As a result, the natural environment is designated as a source of ideas for the special field of design "habitation as an ecological activity" [1].

Appeal to the design methods of harmonization of natural and artificial environment, educational, developmental, and socializing directions are used in a complex way. The specific shades of each of them have a different effect on the student's personality, involving him in successive traditional relationships. In invariant organic design, regional ecological design is an enriching option through the use of an unlimited palette of metaphorical imagery, an individual-cognitive angle of refraction of the "light-energy" (VI. Masalov) potential [3]. The continuous participation of cognitive mechanisms in the process of intensifying creative imagination entails the realization of the relationship between the developing and socializing directions. It implements the unity of the ecological component of culture and landscape heritage (Figure 1). Figure 1 shows the landscapeenvironmental approach of exploratory experimental design.

Traditions as an inheritance of artistic and aesthetic, design, and theoretical achievements are inherent in architectural and design schools and areas, determined by previous development. The socio-historical reasons for innovation lie in the internal laws of artistic development through an experimental approach to project tasks.

The contradiction between the traditional and experimental aspects of design is due either to the dominance of one over the other when traditions lead to formal, dead methods of creativity, or pseudo-experiment completely destroys the design traditions. The development of the "Key" method (Kirienko I. P.) was based on the hypothesis that in invariant organic design, regional ecological design is an enriching option (Figure 2). (Figure 2 shows the hierarchy of cultural " layers "of the regional landscape in the "personlandscape-environment" system). 


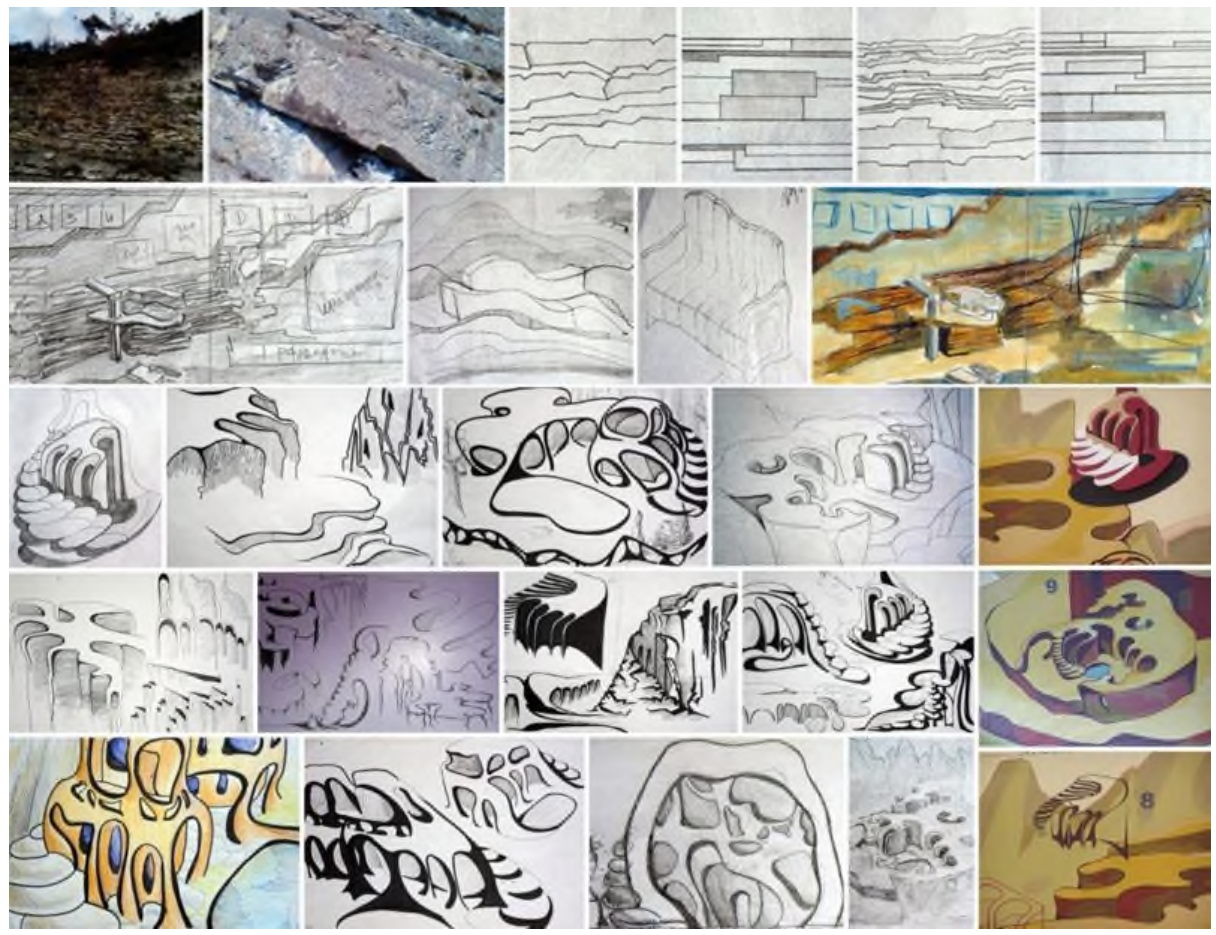

Fig. 1. Transformation of the regional soil structure into a residential design object. Works of students of the 3rd year of SSU K. Khachaturian, Yu. Kuznetsova. Managers: I. P. Kiriyenko, Yu. N. Kiriyenko.

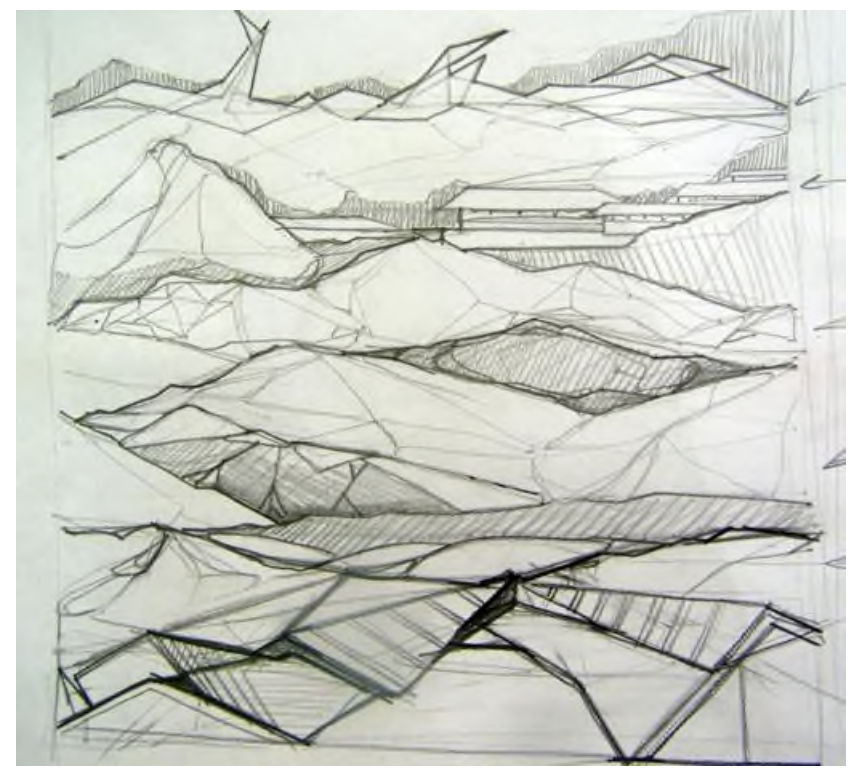

Fig. 2. The birth of the project concept from the landscape. Manual graphics of SSU student L. Matevosyan. Head: I. P. Kiriyenko.

The roots of the alluring mastery of design culture are found in Ancient Greece. Plato and Aristotle are associated with the highest stage of the development of philosophical 
culture, including project culture, which completes the evolution of classical ancient thought.

In the Sochi region, the archaeological, geographical, and historical reality are superimposed on each other in the form of conditions and factors that repeatedly change in space and time, determining the uniqueness of the ways of developing the environmental design. It can be a kind of experimental testing ground for finding innovative solutions: the creative development of "new" environmental objects is harmoniously combined with the monuments of "cultural antiquity" of natural and artificial origin [4]. The virtual design stage is always preceded by manual graphics in all cases involving creativity.

Traditional visual and expressive means, thus, becomes a certain symbol of the mood of the future object. The image arises in the field of feelings during the period of acquaintance with the place of design. The artistry of the image is the prerogative of the easel direction of graphic artists, painters, sculptors. Designers and architects' resort to metaphor, revealing one subject through another, comparing several different independent phenomena [5]. Hand-drawn graphics become a source of imaginative enrichment and a center of attraction for the designer's cognitive mechanisms (Fig. 3). (Figure 3 shows the subject-spatial approach - the search material of the traditional aspect). In addition to "submitting" projects for architectural and design reviews and competitions in the form of computer presentations, designers return to the fact that it is necessary to think "manually" with a "thinking hand" constantly [6].

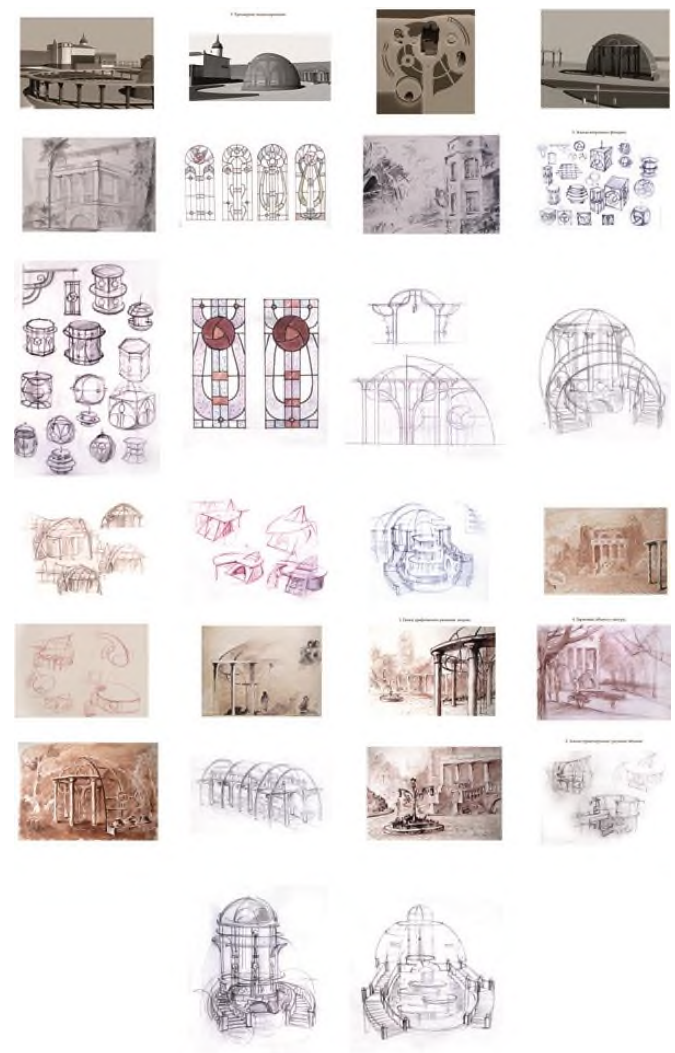

Fig. 3. Preparatory work for the diploma project "Reconstruction of the territory of the villa "Vera" in Sochi". Manual graphics of the SSU student D. Sivakov. The Head of I. P. Kiriyenko. 
Designers "are not obliged to draw beautifully - they must draw intelligently, reveal the movement of space, the development of form in it" [6]. In this creative process, the cognitive mechanisms of design are revealed: in regional design and educational activities, the features of natural materials, their properties, and texture, plastics, and colorists are taken into account, through which informal analogies are" grown " in the developing function of design. The samples of the integrated resort environment create a complex multi-level system, expand the adaptive properties of the resort environment [4].

In educational design, the terrain is used as the basis of the recreational landscape, which determines its compositional structures, the general nature of visual impressions, and to a large extent the functional structure of the territory. The shape of the earth's surface dictates the location of synthetic landscape components, which are largely derived from it. The signs of the design solution must be found in the visibly observed artistic image of natural nature. The organization of the beginning of the creative process, its beginning, deployment, and completion is carried out according to certain rules, which are conventionally represented by the following diagram: highlight key formative elements (lines) - the isolation of their method of abstraction and compositional Association selections or lines in art and design way (research design method "Key") [3].

Interactive components of the natural complexes of the Sochi Black Sea region create "fields of mobility" in the development of previously unsuitable territories for recreational purposes, revealing the possibility of creating original synthetic complexes. On this basis, the pilot projects focus radical revision of the filling of the natural complex of the resort town of aesthetic and environmental sense (Fig. 4). (Figure 4 presents the structural and imaginative approach - an experimental design aspect of a city-resort of Sochi environment).

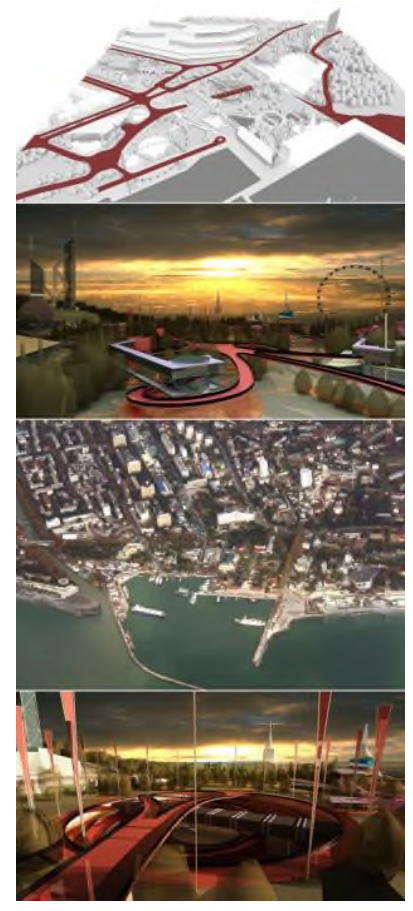

Fig. 4. The design and experimental reconstruction of the territory of the Sochi Seaport are based on the plastic of the natural context. The work of a student of SSU M. Kiriyenko. Heads: I. P. Kiriyenko, E. B. Katrichenko. 


\section{Discussion}

"The architecture reminds me of a chemical reaction in which a product is produced by mixing different elements. You can use a scientific approach, art, literature, music, and even dance. An indispensable ingredient of my kitchen is drawing. This is both a form of presentation of thoughts and the therapy of the body" [6]. This view of graphics confirms the idea of the connection of hand-made architectural and design drawings with design activities, which is expressed in typological forms: travel sketches allow you to explore the historical and cultural heritage of different eras and countries; search sketches allow you to create ideas and to enter the image and design of future architectural design of the object; flow presenter allows you effectively to convey a design idea to the customer; the drawing to the project allows you to clarify the details; a self-contained fantasy graphics allows you to realize your creative potential in the search for new ideas and images. Analog reference points in this variety of manual creativity can serve as drawings by Felten, Quarenghi, and Stackenschneider, graphics by the Archigram group, sketches by Stephen Hall, graphics by Daniel Libeskind, project axonometry by Koolhaas, etchings by Utkin and Brodsky, drawings by Eugene Assa, etc.

But such a point of view does not mean a single view of architectural design, which should overcome the distance between the static idea of form and the dynamic methods of its implementation.

The first creative impulse arises in the cognitive mechanism of the designer on the basis of compliance with the traditional "rule": to convey the main idea of the future object, sometimes a few precise strokes are enough, and the materials and tools themselves suggest the character of the image of the object. The search for a new image under the pencil of an architect-designer determines not only the development of a culture of understanding the form, but also the culture as a whole, including its spiritual aspect. The genre of handdrawn graphics reveals the evolution of the concept and the development of the cognitive mechanisms of design.

The above research design method "Key" [2] is aimed at the development of the bilateral project situation: on the one hand, it provides maximum freedom of developing the creative personality without chilling stamps (experimental aspect), and on the other to create a certain cultural field in space which realizes the talent of the future designer (traditional aspect).

\section{Conclusion}

A variety of opinions can cover both options for abstract-formal harmonization of the subject world (art design) and functionally-motivated proposals for the harmonization of the real subject environment. In both cases, an important theoretical prerequisite is the existence of a comprehensive criterion for assessing the quality of the environment and the possibility of a synthetic approach to assessing beauty and utility in a particular cultural context. "Thus, the hand communicates information to the things it comprehends, and two worlds are formed around the person: the world of "nature", the available, supposed to know things. Not so long ago, it was believed that the history of mankind is a process through which the human hand carries out a progressive transformation of nature into culture" [7].

Based on this, an environmentally sound model of the urban-resort environment in the "person-landscape-environment" system is developed (Fig. 2). The traditional aspect of designing the urban resort environment in the environmental design system is analyzed: landscape-environmental (Fig. 1), subject-spatial (Fig. 3), and structural-figurative aspects (Fig.4). 
The cognitive approach to design is analyzed, when the attitude of self-perception as an integral part of the studied or projected environmental whole is applied. Thus, a model of cognitive mechanisms of design is developed from the standpoint of expanding the emotional palette of the urban resort environment [8].

The complexity of this approach lies in the fact that the criteria of integrity from the standpoint of traditional culture are always rooted in the past (traditional aspect), while the search for similar criteria from the standpoint of technical and technological (civilizational) progress should be directed to the future (experimental aspect). Applying both the traditional and experimental aspects of design, the problem of their coupling with the environmentally sound model "man-landscape-environment" is developed. This, in turn, sets the stage for analyzing and evaluating the cognitive mechanisms of design.

\section{Reference}

1. V. V. Ushnitskiy, IIGU 14 (2015)

2. R. Steiner, The essence of music (Yerevan, Longin, 2010)

3. M. M. Kalinicheva, M. Yu. Kiriyenko, Ecological form formation in the project concept of Rudolf Steiner (Moscow, Designers, 2013)

4. I. P. Kiriyenko, A. I. Novikov, JSFD 2 (2013)

5. I. P. Kiriyenko, VSJ 3, 17 (2011)

6. E. V. Zherdev, BSJ 1, 19 (2012)

7. Archigraphy, The competition of architectural drawing (Moscow, Internet Format, 2014)

8. V. Flusser, Vom Stand der Dinge (Moscow, Ad Mandrian Press, 2016) 